\title{
A SUBCONVEXITY BOUND FOR HECKE $L$-FUNCTIONS
}

\author{
BY ÉTIENNE FOUVRY AND HENRYK IWANIEC ${ }^{1}$
}

ABSTRACT. - Let $K$ be the imaginary quadratic field $\mathbb{Q}(\sqrt{-D})$ with $D$ a prime number congruent to 3 modulo 4 , and let $\psi$ be a Hecke character on $K$ with conductor $(\sqrt{-D})$. Let $L(s, \psi)$ be the corresponding Hecke $L$-function. We prove the upper bound

$$
L(s, \psi)=\mathrm{O}_{\varepsilon}\left(|s|^{\frac{13}{8}} D^{\frac{7}{16}+\varepsilon}\right)
$$

which is valid for every $\varepsilon>0$, uniformly in $D$ and $s$ with $\Re s=\frac{1}{2}$. Note that the exponent in $D$ is strictly less than the exponent $\frac{1}{2}$ which can be deduced classically from the functional equation for $L(s, \psi)$. It is the first result of that type for such $L$-functions.

() 2001 Éditions scientifiques et médicales Elsevier SAS

RÉSUMÉ. - Soit $K$ le corps quadratique imaginaire $\mathbb{Q}(\sqrt{-D})$ avec $D$ un nombre premier congru à 3 modulo 4 , et soit $\psi$ un caractère de Hecke sur $K$, de conducteur $(\sqrt{-D})$. Soit $L(s, \psi)$ la fonction $L$ de Hecke correspondante. On montre la relation

$$
L(s, \psi)=\mathrm{O}_{\varepsilon}\left(|s|^{\frac{13}{8}} D^{\frac{7}{16}+\varepsilon}\right)
$$

qui est valable pour tout $\varepsilon>0$, uniformément en $D$ et en $s$ vérifiant $\Re s=\frac{1}{2}$. Remarquons que l'exposant de $D$ est inférieur à l'exposant $\frac{1}{2}$ qui se déduit, de façon classique, de l'équation fonctionnelle de $L(s, \psi)$. C'est le premier résultat de ce type pour de telles fonctions $L$.

( 2001 Éditions scientifiques et médicales Elsevier SAS

\section{Introduction}

The $L$-functions of different kind (after Dirichlet, Riemann, Hecke, Artin and others) are fascinating subjects of study in contemporary mathematics, because they set up a common ground where arithmetic, geometry and analysis interact strongly and gracefully. Of course, the central problem is the Riemann hypothesis, but there are many important questions which can be addressed and fairly solved without recourse to the Riemann hypothesis. One of these is the order of magnitude of $L$-functions on the critical line in terms of its conductor. If

$$
L(s, f)=\sum_{1}^{\infty} \lambda_{f}(n) n^{-s}
$$

\footnotetext{
${ }^{1}$ Supported in part by NSF Grant DMS-98-01642, the Ambrose Monell Foundation and the Hansmann Membership by a grant to the Institute for Advanced Study. 
with $\lambda_{f}(n) \ll n^{\varepsilon}$ satisfies the functional equation

$$
Q^{s} \Gamma(s+\kappa) L(s, f)=w Q^{1-s} \Gamma(1-s+\kappa) L(1-s, \bar{f})
$$

and some other minor conditions, then by the convexity principle one derives

$$
L(s, f) \ll(|s| Q)^{\alpha+\varepsilon}
$$

on $\Re s=\frac{1}{2}$ with exponent $\alpha=\frac{1}{2}$. The Riemann hypothesis would imply this with $\alpha=0$, however even a slight improvement of the convexity exponent is meaningful for applications (the bound (1.1) with $\alpha=\frac{1}{2}$ is just insufficient, see the survey article by J. Friedlander [4]).

The first subconvexity bound can be traced back to H. Weyl whose method [11] of estimating exponential sums yields

$$
\zeta(s) \ll|s|^{\frac{1}{6}+\varepsilon} .
$$

This bound corresponds to (1.1) for $L(s, f)=\zeta(s)^{2}$ with $\alpha=\frac{1}{3}$. The next example is the bound

$$
L(s, \chi) \ll D^{\frac{3}{16}+\varepsilon}
$$

for Dirichlet characters $\chi(\bmod D)$ which corresponds to (1.1) for $L(s, f)=L(s, \chi)^{2}$ with $\alpha=\frac{3}{8}$ in the $D$-aspect. This result is due to D. Burgess [1]. In this profound work Burgess applied (among some original ideas) the Riemann hypothesis for curves over finite fields. Recently, B. Conrey and H. Iwaniec [2] got

$$
L\left(s, \chi_{D}\right) \ll D^{\frac{1}{6}+\varepsilon}
$$

for the real character $\chi_{D}$ of conductor $D$ by a completely different method (using the nonnegativity of central values of $L$-functions for Maass forms and the Riemann hypothesis for varieties). For another style of $L$-functions W. Duke, J. Friedlander and H. Iwaniec [3] obtained the bound

$$
L(s, f) \ll D^{\frac{1}{4}-\frac{1}{192}+\varepsilon},
$$

where $f$ is a holomorphic, primitive cusp form with respect to the group $\Gamma_{0}(D)$. By now there is a large variety of subconvexity bounds (not only for $L$-functions) many of which are motivated by important consequences.

In this paper we are interested in breaking the convexity bound barrier for

$$
L(s, \psi)=\sum_{0 \neq \mathfrak{a} \subset \mathcal{O}} \psi(\mathfrak{a})(\mathrm{Na})^{-s}
$$

where $\mathcal{O}$ is the ring of integers of an imaginary quadratic field $K=\mathbb{Q}(\sqrt{-D})$ and $\psi$ is a Hecke character of weight $r \geqslant 0$ and conductor $\mathfrak{d}$ which is exactly the different of $K$ (see Section 2 for the notation and a brief review of Hecke theory). These $L$-functions correspond to modular forms on $\Gamma_{0}\left(D^{2}\right)$ with nebentypus character $\chi \chi_{D}$ of modulus $D$. They satisfy the functional equation

$$
\left(\frac{D}{2 \pi}\right)^{s} \Gamma\left(s+\frac{r}{2}\right) L(s, \psi)=w(\psi)\left(\frac{D}{2 \pi}\right)^{1-s} \Gamma\left(1-s+\frac{r}{2}\right) L(1-s, \bar{\psi})
$$

from which it follows that

$$
L(s, \psi) \ll(|s| D)^{\frac{1}{2}+\varepsilon}
$$

$4^{\text {e }}$ SÉRIE - TOME $34-2001-\mathrm{N}^{\circ} 5$ 
(see a direct derivation of a slightly more precise estimate in (6.2)). Our main result is:

THEOREM. - Assume that $D$ is prime, $D \equiv 3(\bmod 4)$ and $\psi(\bmod \mathfrak{d})$ is a Hecke character which on the principal ideals takes values given by $(2.19)$ with $\chi(\bmod D)$ a primitive Dirichlet character. Then for $\Re s=\frac{1}{2}$ we have

$$
L(s, \psi) \ll|s|^{\frac{13}{8}} D^{\frac{7}{16}+\varepsilon}
$$

with any $\varepsilon>0$, the implied constant depending only on $\varepsilon$ and $r$.

Remark. - The hypothesis that $D$ is prime is made exclusively for technical simplifications. Our bound (1.5) is not impressive in the $s$-aspect. We are focused in getting a sharp result in the $D$-aspect, and are satisfied with any bound in terms of $s$ as long as it is of polynomial type. The above exponent $\frac{13}{8}$ crops from crude estimation of our test function. Had we chosen the test function more suitably this exponent could have been lowered considerably (though not without an extra effort). On the other hand the hypothesis that $\psi$ has conductor equal to the different $\mathfrak{d}$ is not entirely made for technical reasons. Without this hypothesis we would arrive at congruences more involved than (5.11), and the present arguments wouldn't work without substantial modifications.

The recent technology for breaking the convexity bounds for $L$-functions goes through averaging over natural companions and by an amplification process of the contribution of the chosen $L$-function. We do not employ the amplification methods, but rather treat the relevant character sums like Burgess (with some new features, see the comments after (5.7)). However, our individual character sums are relatively short so the Burgess' results do not apply directly.

In the special case of trivial nebentypus (i.e. when (2.19) holds with $\chi=\chi_{D}$ ) the old bound (1.2) is applicable giving (1.5) with exponent $\frac{47}{96}$ in place of $\frac{7}{16}$ in the $D$-aspect. However, our Theorem marks the first convexity breaking for automorphic $L$-functions with non-trivial nebentypus characters.

\section{2. $L$-functions for Hecke characters}

In this section we review the Hecke theory in the context of an imaginary quadratic field. Essentially everything that is needed can be found in [5], nevertheless our purpose is to bring these results here in a more explicit form for easy references.

Throughout $K=\mathbb{Q}(\sqrt{-D})$ is the imaginary quadratic field of discriminant $-D$. We assume that $D$ is odd, so $D$ is a positive squarefree number $\equiv 3(\bmod 4)$. Let $\mathcal{O}$ denote the ring of integers of $K$; it is a free $\mathbb{Z}$-module generated by 1 and $\omega=\frac{1}{2}(1+\sqrt{-D})$ :

$$
\mathcal{O}=\mathbb{Z}+\omega \mathbb{Z}=\left\{\frac{1}{2}(m+n \sqrt{-D}) ; m, n \in \mathbb{Z}, m \equiv n(\bmod 2)\right\} .
$$

Next, we assume that $D>3$, so $\mathcal{O}$ has exactly two units, $\mathcal{O}^{*}=\{1,-1\}$. The different of $K$ is the principal ideal $\mathfrak{d}=\sqrt{-D} \mathcal{O}$. The real character $\chi_{D}$ of conductor $D$ given by

$$
\chi_{D}(n)=\left(\frac{-D}{n}\right)=\left(\frac{n}{D}\right)
$$

is called the field character (that the Kronecker symbol equals to the Legendre symbol follows from the quadratic reciprocity law). The value of $\chi_{D}(p)$ determines the type of factorization 
of $p$ into prime ideals in $\mathcal{O}$; we have $p=\mathfrak{p}^{2}, \mathfrak{p}, \mathfrak{p} \overline{\mathfrak{p}}$ with $\mathfrak{p} \neq \overline{\mathfrak{p}}$, if $\chi_{D}(p)=0,-1,1$ respectively. Moreover, the norm of these ideals is $\mathrm{Np}=p, p^{2}, p$ respectively. Hence the zeta function of $K$ factors into

$$
\zeta_{K}(s)=\sum_{0 \neq \mathfrak{a} \subset \mathcal{O}}(\mathrm{Na})^{-s}=\zeta(s) L\left(s, \chi_{D}\right) .
$$

Let $\mathcal{H}=\mathcal{H}(-D)$ and $h=h(-D)$ denote the ideal class group and the class number of $K$. The celebrated Dirichlet class number formula asserts that

$$
h(-D)=\pi^{-1} D^{\frac{1}{2}} L\left(1, \chi_{D}\right) .
$$

On the other hand we have

$$
D^{-\varepsilon} \ll L\left(1, \chi_{D}\right) \ll \log D .
$$

Here the lower bound for $L\left(1, \chi_{D}\right)$ is ineffective (due to C.L. Siegel [10]) while the upper bound is trivial. Therefore

$$
D^{\frac{1}{2}-\varepsilon} \ll h(-D) \ll D^{\frac{1}{2}} \log D .
$$

Every class contains a unique ideal $\mathfrak{a}=\left[a, \frac{1}{2}(b+\sqrt{-D})\right]$ with $a>0, b^{2}-4 a c=-D$ and $(a, b, c)=1$ such that $z_{\mathfrak{a}}=\frac{1}{2 a}(b+\sqrt{-D})$ is in the standard fundamental domain of the modular group; this ideal is called reduced, its norm is $\mathrm{Na}=a$.

The class group $\mathcal{H}$ can be represented as the factor group $I / P$, where $I$ denotes the group of non-zero fractional ideals $\mathfrak{a b}^{-1}$ and $P$ its subgroup of the principal ideals $(\alpha)=\alpha \mathcal{O}$ with $\alpha \in K^{*}$. The dual group $\widehat{\mathcal{H}}$ consists of characters on ideal classes. We shall denote these characters by the letter $\xi$; there are exactly $h$ such characters.

Fix $\mathfrak{m} \subset \mathcal{O}$ a non-zero integral ideal. Let $I_{\mathfrak{m}} \subset I$ be the group of ideals coprime with $\mathfrak{m}$ and

$$
P_{\mathfrak{m}}=\{(\alpha), \alpha \equiv 1(\bmod \mathfrak{m})\}
$$

The factor group $I_{\mathfrak{m}} / P_{\mathfrak{m}}$ is finite, it is called the ray class group.

Fix a non-negative integer $r$ such that $2 \mid r$ if $\mathfrak{m} \mid 2$. A group homomorphism $\psi: I_{\mathfrak{m}} \rightarrow \mathrm{S}^{1}$ satisfying

$$
\psi((\alpha))=\left(\frac{\alpha}{|\alpha|}\right)^{r} \quad \text { if } \alpha \equiv 1(\bmod \mathfrak{m})
$$

is called a Hecke character of modulus $\mathfrak{m}$ and weight $r$. In particular, a Hecke character of modulus $\mathfrak{m}=\mathcal{O}$ and weight $r=0$ is a class group character.

We say that $\psi(\bmod \mathfrak{m})$ is induced by $\psi^{*}\left(\bmod \mathfrak{m}^{*}\right)$ if $\mathfrak{m}^{*} \mid \mathfrak{m}$ and $\psi(\mathfrak{a})=\psi^{*}(\mathfrak{a})$ for every $\mathfrak{a} \in I_{\mathfrak{m}}$. The largest $\mathfrak{m}^{*}$ having this property is called the conductor of $\psi$ and the character $\psi^{*}\left(\bmod \mathfrak{m}^{*}\right)$ is determined by $\psi$ uniquely. Then $\psi(\bmod \mathfrak{m})$ is called primitive if $\mathfrak{m}$ is the conductor of $\psi$. We extend Hecke characters $\psi(\bmod \mathfrak{m})$ to all fractional ideals by setting

$$
\psi(\mathfrak{a})=0 \quad \text { if }(\mathfrak{a}, \mathfrak{m}) \neq \mathcal{O} .
$$

To any Hecke character $\psi(\bmod \mathfrak{m})$ we associate the $L$-function

$$
L(s, \psi)=\sum_{0 \neq \mathfrak{a} \subset \mathcal{O}} \psi(\mathfrak{a})(\mathrm{Na})^{-s}=\prod_{\mathfrak{p}}\left(1-\psi(\mathfrak{p}) \mathrm{N}(\mathfrak{p})^{-s}\right)^{-1} .
$$


Proposition 2.1 (Hecke). - Suppose $\psi(\bmod \mathfrak{m})$ is primitive. Then the function

$$
\Lambda(s, \psi)=(2 \pi)^{-s}(D \mathrm{Nm})^{s / 2} \Gamma\left(s+\frac{r}{2}\right) L(s, \psi)
$$

is entire (except for a simple pole at $s=1$ if $\psi$ is trivial), bounded in vertical strips and it satisfies the functional equation

$$
\Lambda(s, \psi)=w(\psi) \Lambda(1-s, \bar{\psi}) .
$$

The complex number $w(\psi)$ is called the root number. Clearly, (2.11) implies that $w(\psi) w(\bar{\psi})$ $=1$ and $|w(\psi)|=1$. Hecke computed $w(\psi)$ in terms of a Gauss sum $W(\psi)$, precisely

$$
w(\psi)=\mathrm{i}^{-r} W(\psi)(\mathrm{Nm})^{-\frac{1}{2}} .
$$

To define the Gauss sum $W(\psi)$ for the character $\psi(\bmod \mathfrak{m})$, we take an integral ideal $\mathfrak{c}$ in the class of $\mathfrak{m}^{-1}$ such that $(\mathfrak{c}, \mathfrak{m})=\mathcal{O}$ so $\mathfrak{c m}$ is principal. Put $\mathfrak{c m} \mathfrak{d}=(\gamma)$ with $\gamma \in \mathcal{O}$ (recall $\mathfrak{d}$ is the different, and it is a principal ideal). Then

$$
W(\psi)=\sum_{\alpha \in \mathfrak{c} / \mathfrak{c m}} \psi\left(\frac{(\alpha)}{\mathfrak{c}}\right)\left(\frac{\alpha / \gamma}{|\alpha / \gamma|}\right)^{-r} e\left(\operatorname{tr} \frac{\alpha}{\gamma}\right) .
$$

This sum does not depend on the choice of $\mathfrak{c}$ and $\gamma$. If $\psi(\bmod \mathfrak{m})$ is primitive, then $|W(\psi)|=$ $(\mathrm{Nm})^{\frac{1}{2}}$. If $\xi$ is a class group character, then it follows by (2.13) that

$$
W(\xi \psi)=\xi(\mathfrak{m}) W(\psi)
$$

Suppose $\mathfrak{m}$ is principal, say $\mathfrak{m}=\beta \mathcal{O}$ with $\beta \in \mathcal{O}, \beta \neq 0$. Then one can take $\mathfrak{c}=\mathcal{O}$ and $\gamma=\beta \sqrt{-D}$ giving

$$
W(\psi)=\sum_{\alpha \bmod \mathfrak{m}} \psi((\alpha))\left(\frac{\alpha / \gamma}{|\alpha / \gamma|}\right)^{-r} e\left(\operatorname{tr} \frac{\alpha}{\gamma}\right) .
$$

In particular, if $\mathfrak{m}=\mathfrak{d}=\sqrt{-D} \mathcal{O}$, we have $\beta=\sqrt{-D}, \gamma=D$ and (2.15) simplifies to

$$
W(\psi)=\sum_{\alpha \bmod \mathfrak{o}} \psi((\alpha))\left(\frac{\alpha}{|\alpha|}\right)^{-r} e\left(\frac{\alpha+\bar{\alpha}}{\gamma}\right) .
$$

Here all classes $\alpha(\bmod \mathfrak{d})$ are represented by rational integers modulo $D$, so

$$
W(\psi)=\sum_{m \bmod D} \psi((m))\left(\frac{m}{|m|}\right)^{-r} e\left(\frac{2 m}{D}\right) .
$$

The ring inclusion $\mathbb{Z} \subset \mathcal{O}$ defines the isomorphism $\mu: \mathcal{O} / \mathfrak{d} \rightarrow \mathbb{Z} / D \mathbb{Z}$ (because the orders are $|\mathcal{O} / \mathfrak{d}|=\mathrm{N} \mathfrak{d}=D=|\mathbb{Z} / D \mathbb{Z}|)$ given by:

$$
\mu\left(\frac{1}{2}(m+n \sqrt{-D})\right) \equiv \frac{m}{2}(\bmod D)
$$


Hence, for any Dirichlet character $\chi(\bmod D)$ with

$$
\chi(-1)=(-1)^{r}
$$

we get Hecke characters $\psi(\bmod \mathfrak{d})$ such that on all integral principal ideals

$$
\psi((\alpha))=\chi\left(\frac{m}{2}\right)\left(\frac{\alpha}{|\alpha|}\right)^{r}
$$

if $\alpha=\frac{1}{2}(m+n \sqrt{-D}) \in \mathcal{O}$. Of course, if $\chi(\bmod D)$ is primitive as a Dirichlet character then $\psi(\bmod \mathfrak{d})$ is primitive as a Hecke character. Given $\chi(\bmod D)$ there are exactly $h(-D)$ Hecke characters satisfying $(2.19)$, they are obtained by multiplying a fixed $\psi(\bmod \mathfrak{d})$ with the class group characters $\xi$. From now on we are only considering these characters $\xi \psi(\bmod \mathfrak{d})$.

By (2.14) all the characters $\xi \psi$ have the same Gauss sum

$$
W(\psi)=\sum_{m(\bmod D)} \chi(m) e\left(\frac{2 m}{D}\right) .
$$

Hence the root number of the functional equation (2.11) is

$$
w(\psi)=\mathrm{i}^{-r} \bar{\chi}(2) \tau(\chi) D^{-\frac{1}{2}}
$$

where $\tau(\chi)$ is the classical Gauss sum for the Dirichlet character $\chi(\bmod D)$. In particular, if $\chi=\chi_{D}$, then $r$ is odd by (2.18), $\chi_{D}(2)=(-1)^{\frac{D+1}{4}}$ and $\tau\left(\chi_{D}\right)=\mathrm{i} D^{\frac{1}{2}}$ giving

$$
w(\psi)=(-1)^{\frac{r+1}{2}+\frac{D-3}{4}} .
$$

For any character $\psi(\bmod \mathfrak{m})$ we put

$$
\lambda_{\psi}(n)=\sum_{N \mathfrak{a}=n} \psi(\mathfrak{a}) .
$$

Note that $\left|\lambda_{\psi}(n)\right| \leqslant \tau(n)$. If $\psi(\bmod \mathfrak{d})$ is of the type (2.19) then the Fourier series

$$
f_{\psi}(z)=\sum_{n=1}^{\infty} \lambda_{\psi}(n) n^{\frac{r}{2}} e(n z)
$$

gives a primitive cusp form of weight $k=r+1$, level $D^{2}$ and the nebentypus character $\chi_{\chi_{D}}$, i.e. $f_{\psi} \in \mathrm{S}_{k}\left(\Gamma_{0}\left(D^{2}\right), \chi \chi_{D}\right)$. The character $\chi=\chi_{D}$ is special because the resulting nebentypus is trivial. This special case is the subject of studies in series of works by D. Rohrlich, F. RodriguezVillegas and D. Zagier. These authors established numerous results about the central values $L\left(\frac{1}{2}, \psi\right)$ (such as explicit representations in terms of values of theta-series at CM-points, the non-negativity, criteria for the non-vanishing; see, for example, [6-8]).

In this paper we are mainly interested in estimates for $L(s, \psi)$ on the critical line $\Re s=\frac{1}{2}$ for any $\psi(\bmod \mathfrak{d})$ of type $(2.19)$ with the aim to improve the convexity bound in the conductor aspect. 


\section{An approximate functional equation}

In this section we develop an exact formula for $L(s, \psi)$ in terms of two rapidly convergent series. The procedure is quite standard (contour integration of the functional equation) and, when the tails are estimated, the result is often called an approximate functional equation.

Let $G(u)$ be a holomorphic function in the critical strip $-1 \leqslant \Re u \leqslant 1$, such that

$$
\begin{gathered}
G(0)=1, \quad G(u)=G(-u), \\
u^{r+2} G(u) \ll \mathrm{e}^{\frac{\pi}{2}|u|} .
\end{gathered}
$$

Let $\Re s=\frac{1}{2}$. Consider the integral

$$
I(s, \psi)=\frac{1}{2 \pi \mathrm{i}} \int \Lambda(s+u, \psi) G(u) \frac{\mathrm{d} u}{u},
$$

where

$$
\Lambda(s, \psi)=\left(\frac{D}{2 \pi}\right)^{s} \Gamma\left(s+\frac{r}{2}\right) \sum_{1}^{\infty} \lambda_{\psi}(n) n^{-s} .
$$

Moving the line of integration to the line $\Re u=-1$, we pass a simple pole at $u=0$ with residue $\Lambda(s, \psi)$ and we transform the integral on $\Re u=-1$ to that on $\Re u=1$ by applying the functional equation $\Lambda(s, \psi)=w(\psi) \Lambda(1-s, \bar{\psi})$ and changing $s$ into $-s$. We obtain

$$
\Lambda(s, \psi)=I(s, \psi)+w(\psi) I(1-s, \bar{\psi}) .
$$

Next, we compute $I(s, \psi)$ by termwise integration getting

$$
I(s, \psi)=\sum_{1}^{\infty} \lambda_{\psi}(n) \frac{1}{2 \pi \mathrm{i}} \int_{(1)}\left(\frac{D}{2 \pi n}\right)^{s+u} \Gamma\left(s+\frac{r}{2}+u\right) G(u) \frac{\mathrm{d} u}{u} .
$$

Introducing this into the equation above we obtain:

LEMMA 3.1. - For $s$ on the critical line $\Re s=\frac{1}{2}$, we have

$$
L(s, \psi)=\sum_{n=1}^{\infty} \lambda_{\psi}(n) n^{-s} V_{s}\left(\frac{2 \pi n}{D}\right)+w(s, \psi) \sum_{n=1}^{\infty} \lambda_{\bar{\psi}}(n) n^{s-1} V_{1-s}\left(\frac{2 \pi n}{D}\right)
$$

where

$$
\begin{aligned}
& w(s, \psi)=w(\psi)\left(\frac{D}{2 \pi}\right)^{1-2 s} \frac{\Gamma\left(1-s+\frac{r}{2}\right)}{\Gamma\left(s+\frac{r}{2}\right)}, \\
& V_{s}(y)=\frac{1}{2 \pi \mathrm{i}} \int \frac{\Gamma\left(s+\frac{r}{2}+u\right)}{\Gamma\left(s+\frac{r}{2}\right)} \frac{G(u)}{u} y^{-u} \mathrm{~d} u
\end{aligned}
$$

and $G(u)$ is any function which satisfies the conditions (3.1) and (3.2).

Note that $|w(s, \psi)|=1$ for $\Re s=\frac{1}{2}$. 


\section{Properties of the test function $V_{s}(y)$}

In applications of Lemma 3.1 we need to control the growth of $V_{s}(y)$ and its derivatives. To this end we require $G(u)$ to be holomorphic in the strip $-1 \leqslant \Re u \leqslant 1$, such that

$$
u^{3} G(u) \ll \mathrm{e}^{-\frac{\pi}{2}|u|} .
$$

For example we can choose $G(u)=\left(\cos \frac{\pi u}{3}\right)^{-2}$.

LEMMA 4.1. - Let $V_{s}(y)$ be defined by (3.5) with $G(u)$ satisfying (3.1) and (4.1) in the strip $-1 \leqslant \Re u \leqslant 1$. Then, for $s$ with $\Re s=\frac{1}{2}$, we have

$$
y^{\nu} V_{s}^{(\nu)}(y) \ll\left(1+\frac{y}{|s|}\right)^{-1},
$$

for $\nu=0,1,2$, with the implied constant depending only on $r$.

Proof. - In the integral (3.5) we have

$$
\frac{\Gamma\left(s+\frac{r}{2}+u\right)}{\Gamma\left(s+\frac{r}{2}\right)} \ll \frac{(|s|+|u|)^{\frac{r}{2}+1}}{|s|^{\frac{r}{2}}} \mathrm{e}^{-\frac{\pi}{2}|\Im(s+u)|+\frac{\pi}{2}|\Im s|} \ll|s| \mathrm{e}^{\frac{\pi}{2}|u|},
$$

by Stirling's formula. This yields

$$
y^{\nu} V_{s}^{(\nu)}(y) \ll \frac{|s|}{y} \int \mathrm{e}^{\frac{\pi}{2}|u|}\left|G(u) u^{\nu-1}\right||\mathrm{d} u| \ll \frac{|s|}{y} .
$$

Moving the integration to the line $\Re u=-\alpha$ with $0<\alpha<\frac{1}{2}$, we get in the same way that $V_{s}(y)=1+\mathrm{O}\left((y /|s|)^{\alpha}\right)$ and $y^{\nu} V_{s}^{(\nu)}(y) \ll(y /|s|)^{\alpha}$ for $\nu=1,2$, where the first term in $V_{s}(y)$ comes from the simple pole at $u=0$. Combining the above estimates we complete the proof of Lemma 4.1.

Remark. - Assuming the condition (4.1) in a wider strip $-\alpha \leqslant \Re u \leqslant \alpha$ one gets $V_{s}(y) \ll$ $(1+y /|s|)^{-\alpha}$. Hence the series (3.3) can be reduced to $n \leqslant(|s| D)^{1+\varepsilon}$ with $\varepsilon=2 \alpha^{-1}$, the error term being $\mathrm{O}(1 /|s| D)$.

\section{Short character sums}

We shall estimate

$$
S_{\psi}(X)=\sum_{\mathfrak{a}} \psi(\mathfrak{a}) g\left(\frac{\mathrm{Na}}{X}\right),
$$

where $g(y)$ is a smooth function, supported in $[1,2]$, such that

$$
\left|g^{(\nu)}(y)\right| \leqslant P^{\nu}, \quad 0 \leqslant \nu \leqslant 2,
$$

for some $P \geqslant 1$. The trivial bound is

$$
S_{\psi}(X) \ll \sum_{\mathfrak{a}}\left|g\left(\frac{\mathrm{Na}}{X}\right)\right| \ll \sum_{n} \tau(n)\left|g\left(\frac{n}{X}\right)\right| \ll X \log X,
$$


where the constant is absolute. Our goal here is to improve on this, for $X$ satisfying

$$
D^{\frac{1}{2}}<X<D^{\frac{3}{2}}
$$

Let $\mathcal{A}=\{\mathfrak{a}\}$ be the set of representatives of integral ideal classes which consist of ideals $\mathfrak{a}$ such that

$$
\frac{1}{4} \sqrt{\frac{D}{3}}<\mathrm{Na} \leqslant \sqrt{\frac{D}{3}}
$$

These representatives can be constructed out of the reduced ideals $\mathfrak{a}$ by multiplying by $2^{n}$, to ensure that the norm is in the desired interval. Note that every $\mathfrak{a} \in \mathcal{A}$ is prime to $D$ because $D$ is prime.

We split $S_{\psi}(X)$ into classes to get

$$
S_{\psi}(X)=\frac{1}{2} \sum_{\mathfrak{a} \in \mathcal{A}} \psi(\mathfrak{a})^{-1} \sum_{\alpha \equiv 0 \bmod \mathfrak{a}} \psi((\alpha)) g\left(\frac{|\alpha|^{2}}{X N \mathfrak{a}}\right)
$$

where the factor $\frac{1}{2}$ reflects the unit group. Put $\alpha=\frac{1}{2}(m+n \sqrt{-D})$ with $m \equiv n(\bmod 2)$. Inserting (2.19) into (5.3), we get

$$
S_{\psi}(X)=\frac{1}{2} \sum_{\mathfrak{a} \in \mathcal{A}} \psi(\mathfrak{a})^{-1} \sum_{\substack{(m, n) \neq(0,0) \\ m+n \sqrt{-D} \equiv 0 \bmod 2 \mathfrak{a}}} \chi(\overline{2} m)\left(\frac{m+n \sqrt{-D}}{|m+n \sqrt{-D}|}\right)^{r} g\left(\frac{m^{2}+D n^{2}}{4 X N \mathfrak{a}}\right) .
$$

It is now the moment to eliminate from the right side of (5.4) the contribution of the $n=0$. This contribution (of rational ideals) is bounded by

$$
\sum_{\mathfrak{a} \in \mathcal{A}}\left|\left\{0<m<3 X^{\frac{1}{2}} D^{\frac{1}{4}} ; \mathrm{Na} \mid m^{2}\right\}\right| \ll X^{\frac{1}{2}} D^{\frac{1}{4}+\varepsilon}
$$

Hence we obtain

$$
S_{\psi}(X)=\frac{1}{2} S_{\psi}^{*}(X)+\mathrm{O}\left(X^{\frac{1}{2}} D^{\frac{1}{4}+\varepsilon}\right)
$$

where $S_{\psi}^{*}(X)$ is given by (5.4) with the condition $(m, n) \neq(0,0)$ replaced by $n \neq 0$. We simplify the notations by setting $a=\mathrm{Na}$ and $h(z)=(z /|z|)^{r} g\left(|z|^{2}\right)$, and we see that $\left|S_{\psi}^{*}(X)\right| \leqslant T$, where

$$
T=\sum_{\mathfrak{a} \in \mathcal{A}}\left|\sum_{\substack{n \neq 0 \\ m+n \sqrt{-D} \equiv 0 \bmod 2 \mathfrak{a}}} \chi(m) h\left(\frac{m+n \sqrt{-D}}{2 \sqrt{X a}}\right)\right|
$$

By splitting into classes of $m$ modulo $2 a$, we get

$$
T \leqslant \sum_{\mathfrak{a} \in \mathcal{A}} \sum_{n \neq 0} \sum_{\substack{-a<\alpha \leqslant a \\ \alpha+n \sqrt{-D} \equiv 0 \bmod 2 \mathfrak{a}}}\left|\sum_{\ell} \chi(2 a \ell+\alpha) h\left(\frac{2 a \ell+\alpha+n \sqrt{-D}}{2 \sqrt{X a}}\right)\right| .
$$


We shall separate $\ell$ from the other variables by using the Fourier transform of $h(x+\mathrm{i} y)$ in the $x$ variable. We write

$$
h(x+\mathrm{i} y)=\int_{-\infty}^{+\infty} f(v, y) e(v x) \mathrm{d} v
$$

where after Fourier inversion

$$
f(v, y)=\int_{-\infty}^{+\infty} h(x+\mathrm{i} y) e(-v x) \mathrm{d} x \ll\left(1+\frac{|v|}{P}\right)^{-2}
$$

by (5.1) and partial integration. Hence, we get the inequality

$$
T \leqslant \int_{-\infty}^{+\infty} \sum_{\mathfrak{a} \in \mathcal{A}} \sum_{0<|n| \leqslant N} \sum_{\substack{-a<\alpha \leqslant a \\ \alpha+n \sqrt{-D} \equiv 0 \bmod 2 \mathfrak{a}}} \sqrt{X / a}|f(v \sqrt{X / a}, y)|\left|\sum_{|\ell| \leqslant L} \chi(2 a \ell+\alpha) e(v \ell)\right| \mathrm{d} v
$$

with $y=n \sqrt{D / 4 X a}$ and $L=N=4 D^{-\frac{1}{4}} X^{\frac{1}{2}}$ (the above restrictions of the ranges of summations come from the support of $g(z)$, note also that $4 \leqslant L=N \leqslant 4 \sqrt{D}$ ). Hence, for some real $v$, we have

$$
T \ll P \sum_{\mathfrak{a} \in \mathcal{A}} \sum_{0<|n| \leqslant N} \sum_{\substack{-a<\alpha \leqslant a \\ \alpha+n \sqrt{-D} \equiv 0 \bmod 2 \mathfrak{a}}}\left|\sum_{|\ell| \leqslant L} \chi(2 a \ell+\alpha) e(v \ell)\right| .
$$

The innermost sum is a short character sum of length $2 L$ for the Dirichlet character $\chi(\bmod D)$. We need a non-trivial bound for it. Of course, the Riemann hypothesis for $L(s, \chi)$ would help, however we go for unconditional estimates. The difficulty is transparent at the critical value of $X=D$, giving $L=4 D^{\frac{1}{4}}$, which lies below the range of the famous Burgess' result [1]. We shall take advantage of having extra points in the outer summation which enables us to apply the Hölder inequality, without creating a shift in the $\ell$ variable "à la Burgess".

For $x$ modulo $D$, let

$$
\begin{aligned}
\nu(x)=\mid\{(\mathfrak{a}, n, \alpha) ; & \mathfrak{a} \in \mathcal{A}, 0<|n| \leqslant N,-a<\alpha \leqslant a, \\
& \alpha+n \sqrt{-D} \equiv 0 \bmod 2 \mathfrak{a}, \alpha \equiv 2 a x \bmod D\} \mid .
\end{aligned}
$$

With this notation, (5.7) becomes

$$
T \ll P \sum_{x \bmod D} \nu(x)\left|\sum_{|\ell| \leqslant L} \chi(\ell+x) e(v \ell)\right| .
$$

Hölder's inequality implies $T \ll P R_{1}^{\frac{1}{2}} R_{2}^{\frac{1}{4}} V^{\frac{1}{4}}$, with

$$
R_{1}=\sum_{x \bmod D} \nu(x), \quad R_{2}=\sum_{x \bmod D} \nu^{2}(x),
$$


and

$$
V=\sum_{x \bmod D}\left|\sum_{|\ell| \leqslant L} \chi(\ell+x) e(v \ell)\right|^{4}
$$

LEMma 5.1. - We have $R_{1} \ll h(-D) N$.

The proof is easy, it reduces to direct counting of elements of $\mathcal{A}$ and the $n$ 's. Next we prove:

LeMmA 5.2. - For $2 L<D$ we have $V \ll D L^{2}+D^{\frac{1}{2}} L^{4}$.

Proof. - We develop the fourth power into

$$
V \leqslant \sum_{\left|\ell_{1}\right| \leqslant L} \sum_{\left|\ell_{2}\right| \leqslant L} \sum_{\left|\ell_{3}\right| \leqslant L} \sum_{\left|\ell_{4}\right| \leqslant L}\left|\sum_{x} \chi\left(\left(x+\ell_{1}\right)\left(x+\ell_{2}\right)\right) \bar{\chi}\left(\left(x+\ell_{3}\right)\left(x+\ell_{4}\right)\right)\right| .
$$

By Weil's bound for characters sums ([9], Theorem $2 \mathrm{~B}^{\prime}$, p. 43, for instance) we know that the above sum over $x \bmod D$ is $\mathrm{O}\left(D^{\frac{1}{2}}\right)$, provided the rational function

$$
\left(x+\ell_{1}\right)\left(x+\ell_{2}\right) /\left(x+\ell_{3}\right)\left(x+\ell_{4}\right)
$$

is not the $d$ th power of a rational function, where $d$ is the order of $\chi$. If this is a $d$ th power, then $d=2$ and the linear factors are pairwise equal. The number of such cases is bounded by $12 L^{2}$, while the complete sum over $x(\bmod D)$ is trivially estimated by $D$.

The treatment of $R_{2}$ does not require cancellation of terms; nevertheless, it is more delicate than the treatment of $V$. The problem reduces to counting the number of solutions of a system of quadratic congruences in which some variables appear also as moduli.

LEMMA 5.3. - For every positive $\varepsilon$, we have $R_{2} \ll N\left(N+D^{\frac{1}{2}}\right)(D N)^{\varepsilon}$.

Proof. - Clearly, $R_{2}$ is bounded by the number of solutions to the system (see (5.8)):

$$
\left\{\begin{array}{l}
\alpha_{1}^{2}+D n_{1}^{2} \equiv 0\left(\bmod a_{1}\right) \\
\alpha_{2}^{2}+D n_{2}^{2} \equiv 0\left(\bmod a_{2}\right) \\
\alpha_{1} a_{2} \equiv \alpha_{2} a_{1}(\bmod D)
\end{array}\right.
$$

Since we have $a_{1}, a_{2}$ in the segment (5.2) and $\left|\alpha_{1}\right| \leqslant a_{1},\left|\alpha_{2}\right| \leqslant a_{2}$, the last congruence reduces to the equation $\alpha_{1} a_{2}=\alpha_{2} a_{1}$. Putting $d=\left(a_{1}, a_{2}\right), a_{1}=d a_{1}^{*}, a_{2}=d a_{2}^{*}$, we find that the solutions of this equation are

$$
\alpha_{1}=t a_{1}^{*}, \quad \alpha_{2}=t a_{2}^{*}
$$

Note that $|t| \leqslant d \leqslant \sqrt{D}$. Introducing (5.10) into (5.9), we derive the system

$$
\left\{\begin{array}{l}
\alpha_{1}^{2}+D n_{1}^{2} \equiv 0(\bmod d) \\
\alpha_{2}^{2}+D n_{2}^{2} \equiv 0(\bmod d)
\end{array}\right.
$$

and that $a_{1}^{*}\left|n_{1}^{2}, a_{2}^{*}\right| n_{2}^{2}$. We write $a_{1}^{*}=b_{1} c_{1}^{2}, a_{2}^{*}=b_{2} c_{2}^{2}$, with $b_{1}$ and $b_{2}$ squarefree. Recall that $\left(a_{1}^{*}, a_{2}^{*}\right)=1$ so $\left(b_{1} c_{1}, b_{2} c_{2}\right)=1$. Moreover,

$$
n_{1}=b_{1} c_{1} \ell_{1}, \quad n_{2}=b_{2} c_{2} \ell_{2}
$$


with $0<\left|\ell_{1}\right| \leqslant N / b_{1} c_{1}, 0<\left|\ell_{2}\right| \leqslant N / b_{2} c_{2}$. Now (5.11) becomes

$$
\left\{\begin{array}{l}
\left(b_{1} c_{1}\right)^{2}\left(c_{1}^{2} t^{2}+D \ell_{1}^{2}\right) \equiv 0(\bmod d) \\
\left(b_{2} c_{2}\right)^{2}\left(c_{2}^{2} t^{2}+D \ell_{2}^{2}\right) \equiv 0(\bmod d) .
\end{array}\right.
$$

This implies

$$
\left(b_{1} b_{2} c_{1} c_{2}\right)^{2}\left(c_{1}^{2} \ell_{2}^{2}-c_{2}^{2} \ell_{1}^{2}\right) \equiv 0(\bmod d) .
$$

From now on, we consider two cases.

Case 1. -

$$
c_{1}^{2} \ell_{2}^{2} \neq c_{2}^{2} \ell_{1}^{2} \text {. }
$$

We start counting the solutions to (5.13) by fixing $n_{1}, n_{2}$. For given $n_{1}$ and $n_{2}$, the numbers $b_{1}, c_{1}, \ell_{1}, b_{2}, c_{2}, \ell_{2}$ are restricted to the small sets of divisors of $n_{1}$ and $n_{2}$. Moreover, for given $b_{1}, c_{1}, \ell_{1}, b_{2}, c_{2}, \ell_{2}$, the number $d$ is restricted to the small set of divisors of $\left(b_{1} b_{2} c_{1} c_{2}\right)^{2}\left(c_{1}^{2} \ell_{2}^{2}-c_{2}^{2} \ell_{1}^{2}\right)$. Therefore, it remains to estimate the number of solutions of (5.13) in $t(\bmod d)$. We write $d=d_{1} d_{2}$ with $\left(d_{1}, d_{2}\right)=1$ and $\left(d_{1}, b_{1} c_{1}\right)=\left(d_{2}, b_{2} c_{2}\right)=1$. Then $(5.13)$ implies

$$
\left\{\begin{array}{l}
t^{2}+D^{\prime} \ell_{1}^{2} \equiv 0\left(\bmod d_{1}\right), \\
t^{2}+D^{\prime \prime} \ell_{2}^{2} \equiv 0\left(\bmod d_{2}\right),
\end{array}\right.
$$

where $D^{\prime} \equiv D{\overline{c_{1}}}^{2}\left(\bmod d_{1}\right)$ and $D^{\prime \prime} \equiv D{\overline{c_{2}}}^{2}\left(\bmod d_{2}\right)$. This system splits into two independent congruences

$$
\begin{aligned}
t_{1}^{2}+D^{\prime} \ell_{1}^{2} & \equiv 0\left(\bmod d_{1}\right) \\
t_{2}^{2}+D^{\prime \prime} \ell_{2}^{2} & \equiv 0\left(\bmod d_{2}\right) .
\end{aligned}
$$

Hence we get the inequality $|\{t(\bmod d)\}| \leqslant\left|\left\{t_{1}\left(\bmod d_{1}\right)\right\}\right|\left|\left\{t_{2}\left(\bmod d_{2}\right)\right\}\right|$.

LEMMA 5.4. - The number of solutions to

$$
x^{2} \equiv A(\bmod d)
$$

in $x \bmod d$ is bounded by $\tau(d)(d, A)^{\frac{1}{2}}$.

By Lemma 5.4 we get $\left|\left\{t_{1}\left(\bmod d_{1}\right)\right\}\right| \leqslant \tau\left(d_{1}\right)\left(d_{1}, \ell_{1}^{2}\right)^{\frac{1}{2}}$. We have $\left(d_{1}, \ell_{1}^{2}\right) \mid\left(d_{1}, n_{1}^{2}\right)$ and $\left(d_{1}, \ell_{1}^{2}\right) \mid\left(d_{1}, n_{2}^{2}\right)$ because of (5.14). Hence $\left(d_{1}, \ell_{1}^{2}\right) \mid\left(d_{1}, n_{1}^{2}, n_{2}^{2}\right)$. Therefore,

$$
\left|\left\{t_{1}\left(\bmod d_{1}\right)\right\}\right| \leqslant \tau\left(d_{1}\right)\left(d_{1}, n_{1}^{2}, n_{2}^{2}\right)^{\frac{1}{2}}
$$

Multiplying this by the corresponding inequality for $\left|\left\{t_{2}\left(\bmod d_{2}\right)\right\}\right|$ we get

$$
|\{t(\bmod d)\}| \ll \tau(d)\left(n_{1}, n_{2}\right) .
$$

Summing over $n_{1}$ and $n_{2}$, we conclude that the number of solutions to (5.9) in Case 1 is bounded by $\mathrm{O}\left(N^{2}(D N)^{\varepsilon}\right)$.

Case 2.-

$$
c_{1}^{2} \ell_{2}^{2}=c_{2}^{2} \ell_{1}^{2}
$$


Since $\left(c_{1}, c_{2}\right)=1$, we have $\ell_{1}=\lambda c_{1}, \ell_{2}= \pm \lambda c_{2}$ with

$$
0<|\lambda| \leqslant \min \left(\frac{N}{b_{1} c_{1}^{2}}, \frac{N}{b_{2} c_{2}^{2}}\right) \leqslant \frac{N}{c_{1} c_{2} \sqrt{b_{1} b_{2}}} .
$$

In this case, (5.13) yields

$$
\left\{\begin{array}{l}
b_{1}^{2} c_{1}^{4}\left(t^{2}+D \lambda^{2}\right) \equiv 0(\bmod d), \\
b_{2}^{2} c_{2}^{4}\left(t^{2}+D \lambda^{2}\right) \equiv 0(\bmod d) .
\end{array}\right.
$$

Since $\left(b_{1} c_{1}, b_{2} c_{2}\right)=1$, this implies $t^{2}+D \lambda^{2} \equiv 0(\bmod d)$. By Lemma 5.4 the number of solutions in $t(\bmod d)$, is bounded by $\tau(d)\left(d, \lambda^{2}\right)^{\frac{1}{2}} \leqslant \tau(d)(d, \lambda)$. Note that

$$
0<|d| \leqslant \min \left(\frac{\sqrt{D}}{b_{1} c_{1}^{2}}, \frac{\sqrt{D}}{b_{2} c_{2}^{2}}\right) \leqslant \frac{\sqrt{D}}{c_{1} c_{2} \sqrt{b_{1} b_{2}}} .
$$

Finally, summing over $b_{1}, b_{2}, c_{1}, c_{2}, d, \lambda$ we find that the number of solutions to (5.9) in Case 2 is bounded by

$$
\sum_{b_{1}} \sum_{b_{2}} \sum_{c_{1}} \sum_{c_{2}} \sum_{d} \sum_{\lambda} \tau(d)(d, \lambda) \ll N D^{\frac{1}{2}}(D N)^{\varepsilon} .
$$

Adding the results of both cases, we complete the proof of Lemma 5.3.

Putting together Lemmas 5.1, 5.2 and 5.3 we derive under the assumption $D^{\frac{1}{2}}<X<D^{\frac{3}{2}}$, that

$$
S_{\psi}(X) \ll P(h(-D) N)^{\frac{1}{2}}\left(N\left(N+D^{\frac{1}{2}}\right)\right)^{\frac{1}{4}}\left(D L^{2}+D^{\frac{1}{2}} L^{4}\right)^{\frac{1}{4}} D^{\varepsilon}+X^{\frac{1}{2}} D^{\frac{1}{4}+\varepsilon} .
$$

Using now the definition of $L$ and $N$, and the classical upper bound for the class number (2.5), we have

$$
S_{\psi}(X) \ll P D^{\frac{1}{8}} X^{\frac{1}{4}}\left(X^{\frac{1}{2}} D^{-\frac{1}{4}}\left(X^{\frac{1}{2}} D^{-\frac{1}{4}}+D^{\frac{1}{2}}\right)\right)^{\frac{1}{4}}\left(D^{\frac{1}{2}} X+D^{-\frac{1}{2}} X^{2}\right)^{\frac{1}{4}} D^{\varepsilon}+X^{\frac{1}{2}} D^{\frac{1}{4}+\varepsilon} .
$$

Hence we conclude:

Proposition 5.5. - Suppose that $D$ is prime, $D \equiv 3(\bmod 4)$ and that $D^{\frac{1}{2}}<X<D^{\frac{3}{2}}$. Let $g(y)$ be a smooth function supported in $[1,2]$ which satisfies $(5.1)$. Then we have

$$
S_{\psi}(X) \ll P\left(D^{\frac{5}{16}} X^{\frac{5}{8}}+D^{\frac{1}{16}} X^{\frac{7}{8}}\right) D^{\varepsilon} .
$$

Remark. - With no condition on $X$, we claim that

$$
S_{\psi}(X) \ll P\left(D^{\frac{5}{16}} X^{\frac{5}{8}}+D^{\frac{1}{16}} X^{\frac{7}{8}}+D^{-\frac{3}{16}} X^{\frac{9}{8}}\right) D^{\varepsilon}
$$

because this bound is trivial if the conditions of Proposition 5.5 are not satisfied.

\section{Estimating $L(s, \psi)$}

Lemma 3.1 reduces the problem to the estimation of

$$
\nabla(s, \psi)=\sum_{1}^{\infty} \lambda_{\psi}(n) n^{-s} V_{s}\left(\frac{2 \pi n}{D}\right)
$$


because $L(s, \psi)=\nabla(s, \psi)+w(s, \psi) \nabla(1-s, \bar{\psi})$, with $|w(s, \psi)|=1$ on $\Re s=\frac{1}{2}$. Trivially, using $V_{s}(y) \ll(1+y /|s|)^{-1}($ see $(4.2))$, we get

$$
\nabla(s, \psi) \ll \sum_{1}^{\infty} \frac{\tau(n)}{\sqrt{n}}\left(1+\frac{n}{|s| D}\right)^{-1} \ll(|s| D)^{\frac{1}{2}} \log (|s| D) .
$$

Hence

$$
L(s, \psi) \ll(|s| D)^{\frac{1}{2}} \log (|s| D) .
$$

We shall improve on this trivial bound (which is the convexity bound) by applying Proposition 5.5. This requires breaking up $\nabla(s, \psi)$ into partial sums with smooth cut-off functions supported in dyadic segments. We do so by applying a smooth partition of unity

$$
\sum_{\ell=-\infty}^{\infty} Q\left(2^{\frac{\ell}{2}} t\right) \equiv 1, \quad \text { if } t>0
$$

where $Q(t)$ is a smooth function supported in $[1,2]$. Hence $\nabla(s, \psi)=\sum_{\ell} \nabla\left(s, \psi ; 2^{\frac{\ell}{2}}\right)$, where

$$
\nabla(s, \psi ; X)=\sum_{n=1}^{\infty} \lambda_{\psi}(n) n^{-s} V_{s}\left(\frac{2 \pi n}{D}\right) Q\left(\frac{n}{X}\right) .
$$

This is a sum of type $S_{\psi}(X)$ with

$$
g(y)=(y X)^{-s} V_{s}\left(\frac{2 \pi X}{D} y\right) Q(y) .
$$

Using Lemma 4.1 we check that

$$
g^{(\nu)}(y) \ll|s|^{\nu} X^{-\frac{1}{2}}\left(1+\frac{X}{|s| D}\right)^{-1}
$$

for $\nu=0,1,2$. By (5.16) we get

$$
\nabla(s, \psi ; X) \ll D^{\varepsilon}\left(D^{\frac{5}{16}} X^{\frac{5}{8}}+D^{\frac{1}{16}} X^{\frac{7}{8}}+D^{-\frac{3}{16}} X^{\frac{9}{8}}\right)|s| X^{-\frac{1}{2}}\left(1+\frac{X}{|s| D}\right)^{-1} .
$$

Summing over $X=2^{\frac{\ell}{2}} \geqslant \frac{1}{2}$ (the worst value being $X \asymp|s| D$ ) we arrive at

$$
\nabla(s, \psi) \ll|s|^{\frac{13}{8}} D^{\frac{7}{16}+\varepsilon} .
$$

This completes the proof of theorem.

\section{Acknowledgements}

We are grateful to Rutgers and Orsay Universities for hospitality and supporting our exchange of visits when this work was in progress. We also thank the Institute for Advanced Study at Princeton for invitation and support to prepare the results for publication. 


\section{REFERENCES}

[1] Burgess D.A., On character sums and L-series, I, Proc. London Math. Soc. 12 (3) (1962) 193-206.

[2] ConRey J.B, IWANIEC H., The cubic moment of central values of automorphic $L$-functions, Ann. of Math. 151 (2000) 1175-1216.

[3] Duke W., Friedlander J., IWANIEC H., Bounds for automorphic $L$-functions. II, Invent. Math. 115 (1994) 209-217.

[4] Friedlander J., Bounds for $L$-functions, in: Proceedings of the International Congress of Mathematicians, (Zürich, 1994), Birkhäuser Verlag, 1995, pp. 363-373.

[5] Heске E., Eine neue Art von Zetafunktionen und ihre Beziehungen zur Verteilung der Primzahlen, Math. Z. 6 (1920) 11-51.

[6] RodrigueZ Villegas F., On the square root of special values of certain $L$-series, Invent. Math. 106 (1991) 549-573.

[7] Rodriguez Villegas F., Zagier D., Square roots of central values of $L$-series, in: Gouvea F., Yui N. (Eds.), Advances in Number Theory, Proceedings of the Third Conference of the Canadian Number Theory Association, Kingston, Ontario, (1991), Clarendon Press, Oxford, 1993, pp. 81-99.

[8] RoHRLICH D., The non-vanishing of certain Hecke $L$-functions at the center of the critical strip, Duke Math. J. 47 (1980) 223-231.

[9] SCHMidT W., Equations over Finite Fields: An Elementary Approach, Lect. Notes in Math., Vol. 534, Springer-Verlag, 1976.

[10] SiEgel C.L., Über die Classenzahl quadratischer Zahlkörper, Acta Arith. 1 (1936) 83-86.

[11] WeYL H., Zur Abschätzung von $\zeta(1+t i)$, Math. Z. 10 (1921) 88-101.

(Manuscript received November 25, 1999; accepted, after revision, September 29, 2000.)

\footnotetext{
Étienne FOUVRY

Mathématiques, bâtiment 425,

Université de Paris-Sud,

91405 Orsay cedex, France

E-mail: Etienne.Fouvry@math.u-psud.fr

Henryk IWANIEC

Department of Mathematics,

Rutgers University,

New Brunswick, NJ 08405, USA

Current address:

Institute for Advanced Study,

Olden Lane,

Princeton, NJ 08540, USA
} 